\section{Welcher Zwilling bekommt Asthma?}

\author{
Die Unterscheidung, welche Rolle genetische Einflüsse oder \\ Umweltfaktoren bei der Asthmaentwicklung spielen, ist nicht \\ einfach. Einen idealen Ansatz für solche Fragestellungen bietet \\ eine Kontrollstudie mit Zwillingen, von denen jeweils nur einer \\ erkrankt ist.
}

\begin{abstract}
A nhand des dänischen Zwillingsregisters wurden aus den Geburtskohorten der Jahre 1953 bis 1982 insgesamt 6.090 Zwillingspaare ausgewählt, die laut einer 1994 durchgeführten Fragebogenaktion zunächst kein Asthma hatten. In einer Follow-up-Befragung mit vergleichbarer Methodik im Jahr 2002 wurden dann diskordante Paare identifiziert, bei denen inzwischen ein Zwilling Asthma entwickelt hatte. In logistischen Regressionsanalysen wurde nach möglichen Risikofaktoren gesucht.

Von den diskordanten Zwillingspaaren waren 126 monozygot und 273 dizygot. Bei den monozygoten Zwillin-
\end{abstract}

gen waren Heuschnupfen (Odds Ratio [OR] 3,16) und sportliche Betätigung

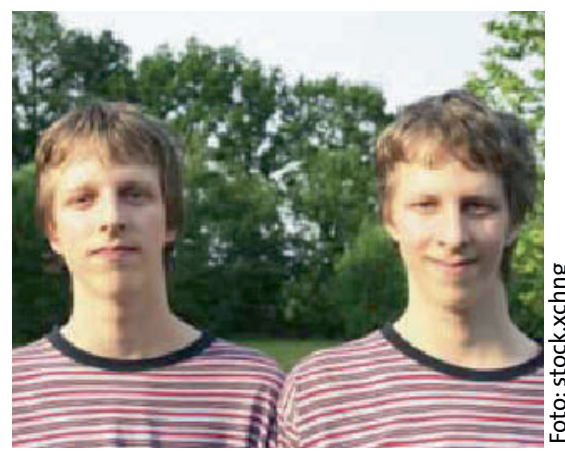

Glaubt man der Statistik, dann hat der sportlichere der beiden Zwillinge ein höheres Asthmarisiko.
(OR 2,86) signifikant mit dem Auftreten von Asthma assoziiert. Sport war dabei definiert als zumindest leichte körperliche Aktivität von wenigstens zwei Stunden pro Woche. Bei den dizygoten Zwillingen erwiesen sich Heuschnupfen (OR 2,44), Ekzeme (OR 1,96), weibliches Geschlecht (OR zwischen Jungen und Mädchen 0,54) und ein zunehmender Body-Mass-Index (OR pro Einheit 1,11 ) als signifikant mit einer Asthmaentwicklung assoziiert.

Fazit: Heuschnupfen, Ekzeme, weibliches Geschlecht, körperliche Aktivität und ein hoher Body-Mass-Index sind dieser Studie zufolge Risikofaktoren für eine Asthmaentwicklung. Das bei monozygoten und dizygoten Zwillingen unterschiedliche Risikoprofil könnte auf eine risikoabhängige genetische $\mathrm{Vul}-$ nerabilität hinweisen.

$b k$

Thomsen SF et al. Risk factors for asthma in young adults: a co-twin control study. Allergy 2006; 61: 229-33

\title{
Kombinierte Strategie bei Asthma
}

\section{Leukotriene und Histamin sind entscheidend an der asthmatischen Frühreaktion auf Inhalationsallergene beteiligt. Zeigt das kom- binierte Blocken beider Mediatoren einen synergistischen Effekt?}

D ie asthmatische Sofortreaktion auf Inhalationsallergene beruht auf der IgE-vermittelten Freisetzung verschiedener Mediatoren durch Mastzellen, die zur Bronchokonstriktion beitragen. Leukotriene und Histamin spielen dabei eine wichtige Rolle. Vor diesem Hintergrund untersuchten kanadische Pneumologen mithilfe der inhalativen Provokation den Effekt einer kombinierten Gabe von $10 \mathrm{mg}$ des Leukotrienantagonisten Montelukast mit 5 mg des Antihistaminikums Desloratadin im Vergleich zur Monotherapie mit den Einzelsubstanzen und zu Plazebo.

Zehn Erwachsene mit leichtem bis mittelschwerem Asthma nahmen an der randomisierten Studie mit vierfachem Crossover-Design teil. Die jeweilige
Studienmedikation wurde 26 und 2 Stunden vor der Provokation gegeben. Die Tests fanden außerhalb der Pollensaison statt. Verwendet wurde jeweils das Allergen, das bei den Probanden im Pricktest die stärkste Reaktion ausgelöst hatte. Zwischen den Provokationen mit den vier unterschiedlichen Medikationen lag stets ein Intervall von mindestens sieben Tagen. Ziel war es, jeweils festzustellen, welche Allergenkonzentration einen 20\%igen Rückgang des FEV1 auslöst $\left(\mathrm{PC}_{20}\right)$.

Unter Plazebo betrug die mittlere $\mathrm{PC}_{20} 104 \mathrm{U} / \mathrm{ml}$, unter Desloratadin alleine $123 \mathrm{U} / \mathrm{ml}$, unter Montelukast alleine $338 \mathrm{U} / \mathrm{ml}$ und bei der Kombination beider Wirkstoffe $697 \mathrm{U} / \mathrm{ml}$. Der Unterschied der Wirksamkeit zwischen der kombinierten Gabe von Montelu- kast und Desloratadin und den Einzelsubstanzen war signifikant $(\mathrm{p}<0,02)$, zu Plazebo sogar hochsignifikant $(\mathrm{p}<$ $0,00001)$. Schließlich war auch die Montelukastwirkung der von Desloratadin und der von Plazebo signifikant überlegen $(p<0,02)$. Der Unterschied der $\mathrm{PC}_{20}$ bei Gabe von Desloratadin oder Plazebo war dagegen nicht statistisch signifikant.

Fazit: Die kombinierte Gabe von Montelukast und Desloratadin hebt bei der inahalativen Provokation die erforderliche Allergendosis zur Auslösung einer asthmatischen Frühreaktion im Vergleich zu Plazebo etwa um den Faktor sieben an. Die Wirkstoffe scheinen in Kombination einen synergistischen Effekt aufzuweisen, der weiterer Untersuchung bedarf.

\section{$f k$}

Davis BE et al. Effect of combined montelukast and desloratadine on the early asthmatic response to inhaled allergen. J Allergy Clin Immunol 2005; 116: 768-72 